\title{
On the Reducibility of Quasiperiodic Linear Hamiltonian Systems and Its Applications in Schrödinger Equation
}

\author{
Nina Xue ${ }^{1}{ }^{1}$ and Wencai Zhao ${ }^{2}$ \\ ${ }^{1}$ School of Mathematics and Information Sciences, Weifang University, Weifang 261061, China \\ ${ }^{2}$ College of Mathematics and Systems Science, Shandong University of Science and Technology, Qingdao, China \\ Correspondence should be addressed to Nina Xue; nnxue20041229@163.com
}

Received 30 January 2020; Accepted 3 April 2020; Published 5 May 2020

Academic Editor: Liguang Wang

Copyright (C) 2020 Nina Xue and Wencai Zhao. This is an open access article distributed under the Creative Commons Attribution License, which permits unrestricted use, distribution, and reproduction in any medium, provided the original work is properly cited.

In this paper, we consider the reducibility of the quasiperiodic linear Hamiltonian system $\dot{x}=(A+\varepsilon Q(t))$, where $A$ is a constant matrix with possible multiple eigenvalues, $Q(t)$ is analytic quasiperiodic with respect to $t$, and $\varepsilon$ is a small parameter. Under some nonresonant conditions, it is proved that, for most sufficiently small $\varepsilon$, the Hamiltonian system can be reduced to a constant coefficient Hamiltonian system by means of a quasiperiodic symplectic change of variables with the same basic frequencies as $Q(t)$. Applications to the Schrödinger equation are also given.

\section{Introduction}

In this paper, we are concerned with the reducibility of the quasiperiodic linear Hamiltonian system

$$
\dot{x}=(A+\varepsilon Q(t)) x, x \in R^{n},
$$

where $A$ is a constant matrix with possible multiple eigenvalues, $Q(t)$ is analytic quasiperiodic with respect to $t$, and $\varepsilon$ is a small parameter.

Firstly, let us recall the definition of the reducibility for quasiperiodic linear systems. Let $A(t)$ be an $n \times n$ quasiperiodic matrix, the differential equation

$$
\dot{x}=A(t) x, \quad x \in R^{n}
$$

is called reducible, if there exists a nonsingular quasiperiodic change of variables

$$
x=\varphi(t) y,
$$

where $\varphi(t)$ and $\varphi^{-1}(t)$ are quasiperiodic and bounded, which changes (2) into

$$
\dot{y}=B y, \quad y \in R^{n},
$$

where $B$ is a constant matrix.

The well-known Floquet theorem states that every periodic differential equation (2) can be reduced to a constant coefficient differential equation (4) by means of a periodic change of variables with the same period as $A(t)$. However, this is not true for the quasiperiodic linear system; one can see [1] for more details. In 1981, Johnson and Sell [2] proved that the quasiperiodic linear system (2) is reducible if the quasiperiodic coefficient matrix $A(t)$ satisfies the "full spectrum" condition.

A typical example of quasiperiodic linear systems comes from the (continuous time) quasiperiodic Schrödinger operators, which are defined on $L^{2}(R)$ as

$$
(L y)(t)=-y^{\prime \prime}(t)+q(\theta+\omega t) y(t),
$$


where $q: T^{n} \longrightarrow R$ is called the potential and $\theta \in T^{n}$ is called the phase. It is well known that the spectrum of $L$ does not depend on the phase when $\omega$ is rationally independent, but it is closely related to the dynamics of the Schrödinger equations

$$
(L y)(t)=-y^{\prime \prime}(t)+q(\theta+\omega t) y(t) E y(t)
$$

or equivalently the dynamics of the linear systems

$$
\dot{x}=V_{E, q}(\theta) x, \quad \dot{\theta}=\omega,
$$

where

$$
V_{E, q}(\theta)=\left(\begin{array}{cc}
0 & 1 \\
q(t)-E & 0
\end{array}\right) \in s l(2, \mathbb{R}) .
$$

Dinaburg and Sinai [3] proved that linear systems (7) are reducible for most $E>E^{*}(q, \alpha, \tau)$, which are sufficiently large, if $\omega$ is fixed and satisfies the Diophantine condition

$$
|\langle k, \omega\rangle| \geq \frac{\alpha}{|k|^{\tau}}, \quad k \in \mathbb{Z}^{r} \backslash\{0\},
$$

where $\alpha, \tau$ are positive constants. The result was generalized by Rüssmann [4] for $\omega$ satisfying the Bruno condition.

Eliasson [5] proved a full measure reducibility result for quasiperiodic linear Schrödinger equations. More precisely, Eliasson proved that (7) is reducible for almost all $E>E *(q, \omega)$ in Lebesgue measure sense, where $\omega$ is a fixed Diophantine vector.

In the case that $n=2$, a stronger reducibility result, called a nonperturbative reducibility, is available. The nonperturbative reducibility means that the smallness of the perturbation does not depend on the Diophantine constant $\alpha$. Hou and You [6] proved, besides other results, the nonperturbative reducibility for (7).

The reducibility of quasiperiodic linear systems with coefficients in $g l(m, R)$ was considered by Jorba and Simó [7]. Suppose that $A$ is a constant matrix with different eigenvalues, they proved that if the eigenvalues of $A$ and the frequencies of $Q$ satisfy some nonresonant conditions, then there exists sufficiently small $\varepsilon_{0}>0$ and a nonempty Cantor set $E \subset\left(0, \varepsilon_{0}\right)$, such that for any $|\varepsilon| \in E$, system (1) is reducible. Moreover, the relative measure of the set $\left(0, \varepsilon_{0}\right) \backslash E$ in $\left(0, \varepsilon_{0}\right)$ is exponentially small in $\varepsilon_{0}$. Junxiang [8] obtained the similar result for the multiple eigenvalue case. Later, many authors [7-10] paid attention to the reducibility of the quasiperiodic linear system (1), which is close to a constant coefficient linear system.

In 1996, Jorba and Simó [10] extended the conclusion of the linear system to the nonlinear system

$$
\dot{x}=(A+\varepsilon Q(t, \varepsilon)) x+\varepsilon g(t)+h(x, t), \quad x \in R^{n} .
$$

Suppose that $A$ has $n$ different nonzero eigenvalues, they proved that under some nonresonant conditions and nondegeneracy conditions, there exists a nonempty Cantor set $E \subset\left(0, \varepsilon_{0}\right)$, such that for all $|\varepsilon| \in E$, system (10) is reducible. Later, Wang and Xu [11] further investigated the nonlinear quasiperiodic system

$$
\dot{x}=A x+f(t, x, \varepsilon), \quad x \in R^{2},
$$

where $A$ is a real $2 \times 2$ constant matrix, and $f(t, 0, \varepsilon)=O(\varepsilon)$, $\partial_{x} f(t, 0, \varepsilon)=O(\varepsilon)$ as $\varepsilon \longrightarrow 0$. They proved without any nondegeneracy condition, one of two results holds: (1) system (11) is reducible to $\dot{y}=B y+O(y)$ for all $\varepsilon \in\left(0, \varepsilon_{0}\right)$; (2) there exists a nonempty Cantor set $E \subset\left(0, \varepsilon_{0}\right)$, such that system (11) is reducible to $\dot{y}=B y+O\left(y^{2}\right)$ for all $|\varepsilon| \in E$.

In [12], Her and You considered one-parameter family of quasiperiodic linear system

$$
\dot{x}=\left(A(\lambda)+g\left(\omega_{1} t, \cdots, \omega_{l} t, \lambda\right)\right) x,
$$

where $A \in C^{\omega}(\Lambda, g l(m, \mathbb{C}))\left(C^{\omega}(\Lambda, \operatorname{gl}(m, \mathbb{C}))\right.$ be the set of $m \times m$ matrices $A(\lambda)$ depending analytically on parameter $\lambda$ in a closed interval $(\Lambda \subset \mathbb{R})$, and $g$ is analytic and small. They proved that under some nonresonant conditions and nondegeneracy conditions, there exists an open and dense set $\mathscr{A}$ in $C^{\omega}(\Lambda, g l(m, \mathbb{C}))$, such that for each $A \in \mathscr{A}$, system (12) is reducible for almost all $\lambda \in \Lambda$.

Instead of a total reduction to a constant coefficient linear system, Jorba et al. [13] investigated the effective reducibility of the following quasiperiodic system:

$$
\dot{x}=(A+\varepsilon Q(t, \varepsilon)) x, \quad|\varepsilon| \leq \varepsilon_{0},
$$

where $A$ is a constant matrix with different eigenvalues. They proved that under nonresonant conditions, by a quasiperiodic transformation, system (13) is reducible to a quasiperiodic system

$$
\dot{y}=\left(A^{*}(\varepsilon)+\varepsilon R^{*}(t, \varepsilon)\right) y, \quad|\varepsilon| \leq \varepsilon_{*} \leq \varepsilon_{0},
$$

where $R^{*}$ is exponentially small in $\varepsilon$. Li and $\mathrm{Xu}$ [14] obtained the similar result for Hamiltonian systems. Later, Xue and Zhao [15] extended the result to the case of multiple eigenvalues.

In this paper, we will study the reducibility of quasiperiodic linear Hamiltonian system (1), where matrix $A$ may have multiple eigenvalues. To this end, the following assumptions are made.

Assumption 1. (nonresonant condition). Let all eigenvalues of matrix $A$ be $\lambda_{1}, \cdots, \lambda_{n}, Q(t)$ be an analytic quasiperiodic function on $D_{\rho}=\left\{\theta \in \mathbb{C}^{r}:\left|\operatorname{Im} \theta_{j}\right| \leq \rho, \bigotimes j=1,2, \cdots, r\right\}$ with the frequencies $\omega=\left(\omega_{1}, \cdots, \omega_{r}\right)$. Suppose that $\lambda=\left(\lambda_{1}, \cdots, \lambda_{n}\right)$ and $\omega=\left(\omega_{1}, \cdots, \omega_{r}\right)$ satisfy the nonresonant conditions

$$
\left|\langle k, \omega\rangle \sqrt{-1}-\lambda_{i}+\lambda_{j}\right| \geq \frac{\alpha}{|k|^{\tau}}
$$

for all $k \in \mathbb{Z}^{r} \backslash\{0\}, 0 \leq i, j \leq n$, where $\alpha>0$ is a small constant and $\tau>r-1$. 
Assumption 2. (nondegeneracy condition). Assume that $A+\varepsilon \bar{Q}$ has $n$ different eigenvalues $\mu_{1}, \cdots, \mu_{n}$ with $\left|\mu_{i}\right| \geq 2 \delta \varepsilon$, $\mu_{i}-\mu_{j} \mid \geq 2 \delta \varepsilon, i \neq j, 0 \leq i, j \leq n$, where $\delta$ is a positive constant independently of $\varepsilon$. Here, we denote the average of $Q(t)$ by $\bar{Q}$, that is,

$$
\bar{Q}=\lim _{T \rightarrow \infty} \frac{1}{2 T} \int_{-T}^{T} Q(t) d t .
$$

We are in a position to state the main result.

Theorem 3. Suppose that Hamiltonian system (1) satisfies Assumptions 1 and 2. Then there exist some sufficiently small $\varepsilon_{0}>0$ and a nonempty Cantor subset $E_{\varepsilon 0} \subset\left(0, \varepsilon_{0}\right)$ with positive Lebesgue measure, such that for $\varepsilon \in E_{\varepsilon 0}$, Hamiltonian system (1) is reducible, i.e., there is an analytic quasiperiodic symplectic transformation $x=\psi(t) y$, where $\psi(t)$ has same frequencies as $Q(t)$, which changes (1) into the Hamiltonian system $\dot{y}=B y$, where $B$ is a constant matrix. Moreover, if $\varepsilon_{0}$ is small enough, the relative measure of $E_{\varepsilon 0}$ in $\left(0, \varepsilon_{0}\right)$ is close to 1 .

Now, we give some remarks on this result. Firstly, here we deal with the Hamiltonian system and have to find the symplectic transformation, which is different from that in $[7,8]$. Secondly, we consider the reducibility, other than the effective reducibility in $[13,14]$. The last but not the least, we can allow matrix $A$ to have multiple eigenvalues. Of course, if the eigenvalues of $A$ are different, the nondegeneracy condition holds naturally.

As an example, we apply Theorem 3 to the following Schrödinger equation:

$$
\ddot{x}+\varepsilon a(t) x=0,
$$

where $a(t)$ is analytic quasiperiodic with the frequencies $\omega=\left(\omega_{1}, \cdots, \omega_{r}\right)$. Denote the average of $a(t)$ by $\bar{a}$. If $\bar{a}>0$ and the frequencies $\omega$ of $a(t)$ satisfy the Diophantine condition

$$
|\langle k, \omega\rangle| \geq \frac{\alpha}{|k|^{\tau}}, \quad k \in \mathbb{Z}^{r} \backslash\{0\}
$$

where $\alpha>0$ is a small constant and $\tau>r-1$, then there exists some sufficiently small $\varepsilon_{0}>0$, equation (17) is reducible and the equilibrium of (17) is stable in the sense of Lyapunov for most sufficiently small $\varepsilon \in\left(0, \varepsilon_{0}\right)$. Moreover, all solutions of equation (17) are quasiperiodic with the frequencies $\Omega=\left(\omega_{1}, \cdots, \omega_{r}, \sqrt{b}\right)$ for most sufficiently small $\varepsilon \in\left(0, \varepsilon_{0}\right)$, where $b=\bar{a} \varepsilon+O\left(\varepsilon^{2}\right)$ as $\varepsilon \longrightarrow 0$. Here, we remark that if we rewrite equation (17) into Hamiltonian system (1), we find that

$$
A=\left(\begin{array}{ll}
0 & 1 \\
0 & 0
\end{array}\right),
$$

which has multiple eigenvalues $\lambda_{1}=\lambda_{2}=0$. One can see Section 4 for more details about this example.

There are plenty of works about the stability of all kinds of equations, one can refer to [16-23] for a detailed description. In particular, for quasiperiodic equations, in order to determine the type of stability of the equilibria of quasiperiodic Hamiltonian systems, the authors need to assume that the corresponding linearized system is reducible, and some conditions were added to the system after the reducibility. However, as far as we know, the case that the conditions are added to the original system has not been considered in the literature up to now, which we will study in the future.

The paper is organized as follows. In Section 2, we list some basic definitions and results that will be useful in the proof of the main result. In Section 3, we will prove Theorem 3. Equation (17) will be analyzed in Section 4.

\section{Some Preliminaries}

We first give the definition of quasiperiodic functions.

Definition 4. A function $f$ is said to be a quasiperiodic function with a vector of basic frequencies $\omega=\left(\omega_{1}, \omega_{2}, \cdots, \omega_{r}\right)$, if $f(t)=F\left(\theta_{1}, \theta_{2}, \cdots, \theta_{r}\right)$, where $F$ is $2 \pi$ periodic in all its arguments and $\theta_{j}=\omega_{j} t$ for $j=1,2, \cdots, r$. Moreover, if $F(\theta)$ $\left(\theta=\left(\theta_{1}, \theta_{2}, \cdots, \theta_{r}\right)\right)$ is analytic on $D_{\rho}=\left\{\theta \in \mathbb{C}^{r}:\left|\operatorname{Im} \theta_{j}\right|\right.$ $\leq \rho, j=1,2, \cdots, r\}$, we say that $f(t)$ is analytic quasiperiodic on $D_{\rho}$.

It is well known that an analytic quasiperiodic function $f(t)$ can be expanded as Fourier series

$$
f(t)=\sum_{k \in \mathbb{Z}^{r}} f_{k} e^{\langle k, \omega\rangle \sqrt{-1} t},
$$

with Fourier coefficients defined by

$$
f_{k}=\frac{1}{(2 \pi)^{r}} \int_{T^{r}} F(\theta) e^{-\langle k, \theta\rangle \sqrt{-1}} d \theta .
$$

Denote by $\|f\|_{\rho}$ the norm

$$
\|f\|_{\rho}=\sum_{k \in \mathbb{Z}^{r}}\left|f_{k}\right| e^{|k| \rho} .
$$

Definition 5. An $n \times n$ matrix $Q(t)=\left(q_{i j}(t)\right)_{1 \leq i, j \leq n}$ is said to be analytic quasiperiodic on $D_{\rho}$ with frequencies $\omega=\left(\omega_{1}, \omega_{2}\right.$, $\left.\cdots, \omega_{r}\right)$, if all $q_{i j}(t)(i, j=1,2, \cdots, n)$ are analytic quasiperiodic on $D_{\rho}$ with frequencies $\omega=\left(\omega_{1}, \omega_{2}, \cdots, \omega_{r}\right)$.

Define the norm of $Q$ by

$$
\|Q\|_{\rho}=\max _{1 \leq i \leq n} \sum_{j=1}^{n}\left\|q_{i j}\right\|_{\rho} .
$$


It is easy to see that

$$
\left\|Q_{1} Q_{2}\right\|_{\rho} \leq\left\|Q_{1}\right\|_{\rho}\left\|Q_{2}\right\|_{\rho}
$$

If $Q$ is a constant matrix, write $\|Q\|=\|Q\|_{\rho}$ for simplicity. Denote the average of $Q(t)$ by $\bar{Q}=\left(\bar{q}_{i j}\right)_{1 \leq i, j \leq n}$, where

$$
\bar{q}_{i j}=\lim _{T \rightarrow \infty} \frac{1}{2 T} \int_{-T}^{T} q_{i j}(t) d t
$$

See [24] for the existence of the limit.

Also, we need two lemmas which are provided in this section for the proof of Theorem 3 that were proved in [10].

Lemma 6. Let $h: B_{\sigma}(0) \subset \mathbb{R}^{n} \longrightarrow \mathbb{R}^{n}$ be a $C^{2}$ function that satisfies $h(0)=0, \quad D_{x} h(0)=0, \quad\left\|D_{x x} h(x)\right\| \leq K, \quad x \in B_{\sigma}(0)$. Then, $\|h(x)\| \leq K / 2\|x\|^{2},\left\|D_{x} h(x)\right\| \leq K\|x\|$.

Lemma 7. Suppose that $B_{0}$ is an $n \times n$ matrix with different nonzero eigenvalues satisfying $\left|\mu_{i}^{0}\right|>\gamma,\left|\mu_{i}^{0}-\mu_{j}^{0}\right|>\gamma, i \neq j$, $1 \leq i, j \leq n$, and $S_{0}$ is a regular matrix such that $S_{0}^{-1} B_{0} S_{0}=$ $\operatorname{diag}\left(\mu_{1}^{0}, \cdots, \mu_{n}^{0}\right)$. Set $\beta_{0}=\max \left\{\left\|S_{0}\right\|,\left\|S_{0}^{-1}\right\|\right\}$, and choose $b$ such that

$$
0<b<\frac{\gamma}{(3 n-1) \beta_{0}^{2}}
$$
hold:

If $B_{1}$ verifies $\left\|B_{1}-B_{0}\right\| \leq b$, then the following conclusions

(1) $B_{1}$ has $n$ different nonzero eigenvalues $\mu_{1}^{1}, \cdots, \mu_{n}^{1}$

(2) There exists a regular matrix $S_{1}$ such that $S_{1}^{-1} B_{1} S_{1}=$ $\operatorname{diag}\left(\mu_{1}^{1}, \cdots, \mu_{n}^{1}\right)$, which satisfies $\|S\|,\left\|S_{1}^{-1}\right\| \leq \beta_{1}$, where $\beta_{1}=2 \beta_{0}$.

The next lemma is used to perform a step of the inductive procedure in the proof of Theorem 3.

Lemma 8. Consider the differential equation

$$
\dot{P}(t)=\Lambda P(t)-P(t) \Lambda+R(t)
$$

where $\Lambda$ is a constant Hamiltonian matrix with $n$ different eigenvalues $v_{1}, \cdots, v_{n}, R$ is an analytic quasiperiodic Hamiltonian matrix on $D_{\rho}$ with frequencies $\omega$ satisfying $\bar{R}=0$.

If

$$
\left|\langle k, \omega\rangle \sqrt{-1}-v_{i}+v_{j}\right| \geq \frac{\alpha}{|k|^{3 \tau}}
$$

for all $0 \neq k \in \mathbb{Z}^{r}$, and $\left|v_{i}\right| \geq \delta \varepsilon,\left|v_{i}-v_{j}\right| \geq \delta \varepsilon$, for $i \neq j, 0 \leq i$, $j \leq n$, where $\delta$ is a positive constant independent of $\varepsilon$, then equation (27) has a unique analytic quasiperiodic Hamiltonian solution $P(t)$ with $\bar{P}=0$, where $P(t)$ has frequencies $\omega$ and satisfies

$$
\|P\|_{\rho-s} \leq \frac{c}{\alpha s^{v}}\|R\|_{\rho}
$$

with $v=3 \tau+r$ and $0<s<\rho$, where the constant $c$ depends only on $\tau$ and $r$.

Proof. Choosing $S$ such that $S^{-1} \Lambda S=D=\operatorname{diag}\left(v_{1}, \cdots, v_{n}\right)$, making the change of variable $P(t)=S X(t) S^{-1}$ and defining $Y(t)=S^{-1} R(t) S$, equation (27) becomes

$$
\dot{X}(t)=D X(t)-X(t) D+Y(t), \quad \bar{Y}=0 .
$$

Expanding $X$ and $Y$ into Fourier series yields that

$$
\begin{aligned}
& X(t)=\sum_{k \in \mathbb{Z}^{r}} X_{k} e^{\langle k, \omega\rangle \sqrt{-1} t}, \\
& Y(t)=\sum_{k \in \mathbb{Z}^{r}} Y_{k} e^{\langle k, \omega\rangle \sqrt{-1} t},
\end{aligned}
$$

where $X_{k}=\left(x_{i j}^{k}\right)_{1 \leq i, j \leq n}$ and $Y_{k}=\left(y_{i j}^{k}\right)_{1 \leq i, j \leq n}$.

By comparing the coefficients of (30), we obtain that

$$
x_{i j}^{k}=\frac{y_{i j}^{k}}{\langle k, \omega\rangle \sqrt{-1}-v_{i}+v_{j}}, \quad 1 \leq i, j \leq n, k \neq 0,
$$

$$
x_{0}^{i j}=0, \quad 1 \leq i, j \leq n .
$$

Since $R$ is analytic on $D_{\rho}, Y$ is also analytic on $D_{\rho}$. Therefore, we have

$$
\left\|Y_{k}\right\| \leq\|Y\|_{\rho} e^{-|k| \rho}
$$

Hence,

$$
\|X\|_{\rho-s}=\sum_{k \in \mathbb{Z}^{r}}\left\|X_{k}\right\| e^{|k|(\rho-s)} \leq \sum_{0 \neq k \in \mathbb{Z}^{r}} \frac{|k|^{3 \tau} e^{-s|k|}}{\alpha}\|Y\|_{\rho} \leq \frac{c}{\alpha s^{v}}\|Y\|_{\rho},
$$

where $v=3 \tau+r$ and $0<s<\rho$. Here and hereafter, we always use the same symbol $c$ to denote different constants in estimates. Hence,

$$
\|P\|_{\rho-s} \leq c\|X\|_{\rho-s} \leq \frac{c}{\alpha s^{v}}\|Y\|_{\rho} \leq \frac{c}{\alpha s^{v}}\|R\|_{\rho} .
$$

Now, we prove that $P$ is Hamiltonian. Since $\Lambda$ and $R$ are Hamiltonian, then $\Lambda=J \Lambda_{J}$ and $R=J R_{J}$, where $\Lambda_{J}$ and $R_{J}$ are symmetric. Let $P_{J}=J^{-1} P$, if $P_{J}$ is symmetric, then $P$ is Hamiltonian. Below, we prove that $P_{J}$ is symmetric. 
Substituting $P=J P_{I}$ into equation (27) yields

$$
\dot{P}_{J}=\Lambda_{J} J_{P}^{J}-P_{J} J \Lambda_{J}+R_{J}
$$

and transposing equation (37), we get

$$
\dot{P}_{J}^{T}=\Lambda_{J} J P_{J}^{T}-P_{J}^{T} J \Lambda_{J}+R_{J}
$$

It is easy to see that $J P_{J}$ and $J P_{J}^{T}$ are solutions of (27); moreover, $\overline{J P_{J}}=\overline{J P_{J}^{T}}=0$. Since the solution of (27) with $\bar{P}=0$ is unique, we have $J P_{J}=J P_{J}^{T}$, which implies that $P$ is Hamiltonian. Up to now, we have finished the proof of this lemma.

\section{Proof of Theorem 3}

From the assumptions of Theorem 3, it follows that $A+\varepsilon \bar{Q}$ is a Hamiltonian matrix with $n$ different eigenvalues $\mu_{1}, \cdots, \mu_{n}$, and $\left|\mu_{i}\right| \geq 2 \delta \varepsilon,\left|\mu_{i}-\mu_{j}\right| \geq 2 \delta \varepsilon, i \neq j, 0 \leq i, j \leq n$, where $\delta$ is a positive constant independent of $\varepsilon$. We rewrite Hamiltonian system (1) into

$$
\dot{x}=[A+\varepsilon \bar{Q}+\varepsilon(Q(t)-\bar{Q})] x:=\left(A_{1}+\varepsilon \tilde{Q}(t)\right) x,
$$

where $A_{1}=A+\varepsilon \bar{Q}, \tilde{Q}(t)=Q(t)-Q$, and $\tilde{Q}(t)=0$.

Introduce the change of variables $x=e^{\varepsilon P(t)} x_{1}$, where $P(t)$ will be determined later, under this symplectic transformation, Hamiltonian system (39) is changed into the new Hamiltonian system

$$
\dot{x}_{1}=e^{-\varepsilon P(t)}\left(A_{1}+\varepsilon \tilde{Q}-\varepsilon \dot{P}\right) e^{\varepsilon P(t)} x_{1} .
$$

Expand $e^{\varepsilon P}$ and $e^{-\varepsilon P}$ into

$$
\begin{aligned}
e^{\varepsilon P} & =I+\varepsilon P+B, \\
e^{-\varepsilon P} & =I-\varepsilon P+\tilde{B},
\end{aligned}
$$

where

$$
\begin{aligned}
& B=\frac{(\varepsilon P)^{2}}{2 !}+\frac{(\varepsilon P)^{3}}{3 !}+\cdots, \\
& \tilde{B}=\frac{(\varepsilon P)^{2}}{2 !}-\frac{(\varepsilon P)^{3}}{3 !}+\cdots
\end{aligned}
$$

Then, system (40) can be rewritten

$$
\begin{aligned}
\dot{x}_{1} & =(I-\varepsilon P+\tilde{B})\left(A_{1}+\varepsilon \tilde{Q}-\varepsilon \dot{P}\right)(I+\varepsilon P+B) x_{1} \\
& =\left(A_{1}+\varepsilon \tilde{Q}-\varepsilon \dot{P}+\varepsilon A_{1} P-\varepsilon P A_{1}+\varepsilon^{2} Q_{1}\right) x_{1}
\end{aligned}
$$

where

$$
\begin{aligned}
Q_{1}= & -P(\tilde{Q}-\dot{P})+(\tilde{Q}-\dot{P}) P-P\left(A_{1}+\varepsilon \tilde{Q}-\varepsilon \dot{P}\right) P+(I-\varepsilon P) \\
& \cdot\left(A_{1}+\varepsilon \tilde{Q}-\varepsilon \dot{P}\right) \frac{B}{\varepsilon^{2}}+e^{\varepsilon P}\left(A_{1}+\varepsilon \tilde{Q}-\varepsilon \dot{P}\right) \frac{\tilde{B}}{\varepsilon^{2}}
\end{aligned}
$$

We would like to have

$$
\tilde{Q}-\dot{P}+A_{1} P-P A_{1}=0,
$$

which is equivalent to

$$
\dot{P}=A_{1} P-P A_{1}+\tilde{Q}
$$

By Assumption 2 of Theorem 3, it is easy to see that the inequalities

$$
\left|\mu_{i}\right| \geq \delta \varepsilon,\left|\mu_{i}-\mu_{j}\right| \geq \delta \varepsilon, \quad i \neq j, 0 \leq i, j \leq n
$$

hold. Moreover, if the equalities

$$
\left|\langle k, \omega\rangle \sqrt{-1}-\mu_{i}+\mu_{j}\right| \geq \frac{\alpha_{0}}{|k|^{3 \tau}}, \quad 0 \neq k \in \mathbb{Z}^{r},
$$

also hold, where $\alpha_{0}=\alpha / 2$, thus, by Lemma 8 , (46) is solvable for $P$ on a smaller domain, that is, there is a unique quasiperiodic Hamiltonian matrix $P(t)$ with frequencies $\omega$ on $D_{\rho-s}$, which satisfies $\bar{P}=0$ and

$$
\|P\|_{\rho-s} \leq \frac{c}{\alpha_{0} s^{v}}\|\tilde{Q}\|_{\rho} \leq \frac{c}{\alpha_{0} s^{v}}\|Q\|_{\rho}
$$

where $s=(1 / 2) \rho$.Therefore, by (46), Hamiltonian system (43) becomes

$$
\dot{x}_{1}=\left(A_{1}+\varepsilon^{2} Q_{1}\right) x_{1},
$$

where

$$
\begin{aligned}
Q_{1}= & P\left(A_{1} P-P A_{1}\right)+\left(P A_{1}-A_{1} P\right) P-P\left(A_{1}-\varepsilon\left(P A_{1}-A_{1} P\right)\right) \\
& \cdot P+(I-\varepsilon P)\left(A_{1}-\varepsilon\left(P A_{1}-A_{1} P\right)\right) \frac{B}{\varepsilon^{2}} \\
& +\frac{\tilde{B}}{\varepsilon^{2}}\left(A_{1}-\varepsilon\left(P A_{1}-A_{1} P\right)\right) e^{\varepsilon P} .
\end{aligned}
$$

From Lemma 6, it follows that

$$
\begin{aligned}
& \|B\|_{\rho-s} \leq c\|\varepsilon P\|_{\rho-s}^{2}, \\
& \|\tilde{B}\|_{\rho-s} \leq c\|\varepsilon P\|_{\rho-s}^{2} .
\end{aligned}
$$


Therefore, if $\varepsilon$ is sufficiently small, we have

$$
\left\|Q_{1}\right\|_{\rho-s} \leq c\|P\|_{\rho-s}^{2} \leq \frac{c}{\alpha_{0}^{2} s^{2 v}}\|Q\|_{\rho}^{2}
$$

Now we consider the iteration step. In the $m^{\text {th }}$ step, we consider the Hamiltonian system

$$
\dot{x}_{m}=\left(A_{m}+\varepsilon^{2 m} Q_{m}(t)\right) x_{m}, m \geq 1,
$$

where $A_{m}$ has $n$ different eigenvalues $\lambda_{1}^{m}, \cdots, \lambda_{n}^{m}$ with

$$
\begin{aligned}
\left|\lambda_{i}^{m}\right| & \geq \delta \varepsilon, \\
\left|\lambda_{i}^{m}-\lambda_{j}^{m}\right| & \geq \delta \varepsilon, \\
i \neq j, 1 & \leq i, j \leq n .
\end{aligned}
$$

Here, we define $\lambda_{i}^{1}=\mu_{i}, i=1, \cdots, n$.

Let $A_{m+1}=A_{m}+\varepsilon^{2 m} \bar{Q}_{m}$, then system (54) becomes

$$
\dot{x}_{m}=\left(A_{m+1}+\varepsilon^{2 m} \tilde{Q}_{m}\right) x_{m}, \quad m \geq 1,
$$

where $\tilde{Q}_{m}=Q_{m}(t)-\bar{Q}_{m}$. We need to solve

$$
\dot{P}_{m}=A_{m+1} P_{m}-P_{m} A_{m+1}+\tilde{Q}_{m}
$$

If

$$
\left|\langle k, \omega\rangle \sqrt{-1}-\lambda_{i}^{m+1}+\lambda_{j}^{m+1}\right| \geq \frac{\alpha_{m}}{|k|^{3 \tau}}, \quad 0 \neq k \in \mathbb{Z}^{r},
$$

and $A_{m+1}$ has $n$ different eigenvalues $\lambda_{1}^{m+1}, \cdots, \lambda_{n}^{m+1}$ with

$$
\begin{aligned}
&\left|\lambda_{i}^{m+1}\right| \geq \delta \varepsilon, \\
&\left|\lambda_{i}^{m+1}-\lambda_{j}^{m+1}\right| \geq \delta \varepsilon, \\
& i \neq j, 1 \leq i, j \leq n,
\end{aligned}
$$

by Lemma 8 , there is a unique quasiperiodic Hamiltonian matrix $P_{m}(t)$ with frequencies $\omega$ on $D_{\rho_{m}-s_{m}}$, which satisfies

$$
\left\|P_{m}\right\|_{\rho_{m}-s_{m}} \leq \frac{c}{\alpha_{m} s_{m}^{v}}\left\|Q_{m}\right\|_{\rho_{m}}
$$

Thus, under the symplectic change of variables $x_{m}=e^{\varepsilon^{2 m} P_{m}(t)} x_{m+1}$, system (56) is changed into

$$
\dot{x}_{m+1}=\left(A_{m+1}+\varepsilon^{2^{m+1}} Q_{m+1}\right) x_{m+1},
$$

where

$$
\begin{aligned}
Q_{m+1}(t)= & P_{m}\left(A_{m+1} P_{m}-P_{m} A_{m+1}\right) \\
& +\left(P_{m} A_{m+1}-A_{m+1} P_{m}\right) P_{m} \\
& -P_{m}\left(A_{m+1}+\varepsilon^{2 m}\left(P_{m} A_{m+1}-A_{m+1} P_{m}\right)\right) P_{m} \\
& +\left(I-\varepsilon^{2 m} P_{m}\right)\left(A_{m+1}+\varepsilon^{2 m}\left(P_{m} A_{m+1}-A_{m+1} P_{m}\right)\right) \frac{B_{m}}{\varepsilon^{2 m+1}} \\
& +\frac{\tilde{B}_{m}}{\varepsilon^{2 m+1}}\left(A_{m+1}+\varepsilon^{2 m}\left(P_{m} A_{m+1}-A_{m+1} P_{m}\right)\right) \varepsilon^{2 m} P_{m}, e^{-\varepsilon^{2^{m}} P_{m}} \\
= & I+\varepsilon^{2^{m}} P_{m}+B_{m}, e^{-\varepsilon^{2^{m}} P_{m}}=I-\varepsilon^{2^{m}} P_{m}+\tilde{B}_{m}, \\
B_{m}= & \frac{\left(\varepsilon^{2^{m}} P_{m}\right)^{2}}{2 !}+\frac{\left(\varepsilon^{2^{m}} P_{m}\right)^{3}}{3 !}+\cdots, \\
\tilde{B} m= & \frac{\left(\varepsilon^{2^{m}} P_{m}\right)^{2}}{2 !}+\frac{\left(\varepsilon^{2^{m}} P_{m}\right)^{3}}{3 !}+\cdots .
\end{aligned}
$$

From Lemma 6, it follows that

$$
\begin{aligned}
& \left\|B_{m}\right\|_{\rho_{m}-s_{m}} \leq c\left\|\varepsilon^{2^{m}} P_{m}\right\|_{\rho_{m}-s_{m}}^{2}, \\
& \left\|\tilde{B}_{m}\right\|_{\rho_{m}-s_{m}} \leq c\left\|\varepsilon^{2^{m}} P_{m}\right\|_{\rho_{m}-s_{m}}^{2} .
\end{aligned}
$$

Therefore, if $|\varepsilon|$ is sufficiently small, by (60) we have

$$
\left\|Q_{m+1}\right\|_{\rho_{m}-s_{m}} \leq \frac{c}{\alpha_{m}^{2} s_{m}^{2 v}}\left\|Q_{m}\right\|_{\rho_{m}}^{2}
$$

Now we prove that the iteration is convergent as $m \longrightarrow \infty$. When $m=1$, we choose

$$
\begin{aligned}
\alpha_{1} & =\frac{1}{4} \alpha, \\
\rho_{1} & =\frac{1}{2} \rho, \\
s_{1} & =\frac{1}{8} \rho, \\
F_{1} & =\frac{\left\|\varepsilon^{2} Q_{1}\right\|_{\rho 1}}{\alpha_{1}^{2} s_{1}^{2 v}} .
\end{aligned}
$$

At the $m^{\text {th }}$ step, we define

$$
\begin{aligned}
\alpha_{m} & =\frac{\alpha}{(m+1)^{2}}, \\
s_{m} & =\frac{\rho}{2^{m+2}}, \\
\rho_{m} & =\rho_{1}-\left(s_{1}+s_{2}+\cdots+s_{m-1}\right) \\
F_{m} & =\frac{\varepsilon^{2^{m}}\left\|Q_{m}\right\|_{\rho_{m}}}{\alpha_{m}^{2} s_{m}^{2 v}} .
\end{aligned}
$$


By (64), we have

$$
F_{m+1} \leq c \frac{\mathcal{E}^{2^{m+1}}\left\|Q_{m}\right\|_{\rho_{m}}^{2}}{\left(\alpha_{m}^{2} s_{m}^{2 v}\right)^{2}}=c F_{m}^{2},
$$

where the constant $c$ depends only on $\alpha, \rho$. Hence, it follows that

$$
c F_{m+1} \leq\left(c F_{m}\right)^{2} \leq\left(c F_{1}\right) 2^{m} .
$$

If $c F_{1}<1$, then $c F_{m} \longrightarrow 0$ as $m \longrightarrow \infty$. From (60), it follows that

$$
\left\|\varepsilon^{2^{m}} P_{m}\right\|_{\rho_{m}-s_{m}}<c F_{m} .
$$

Thus, if $c F_{1}<1 / 2$, then

$$
\left\|e^{ \pm \varepsilon^{2^{m}}} P_{m}\right\|_{\rho_{m}} \leq 2
$$

Since

$$
\left\|A_{m+1}-A_{m}\right\|=\left\|\varepsilon^{2^{m}} \bar{Q}_{m}\right\| \leq\left\|\varepsilon^{2^{m}} Q_{m}\right\|_{\rho_{m}}<c F_{m}
$$

if $c F_{1} \leq \delta \varepsilon /(3 n-1) \beta_{m}^{2}$, it follows from (71) that

$$
\left\|A_{m+1}-A_{m}\right\| \leq \frac{\delta \varepsilon}{(3 n-1) \beta_{m}^{2}}, \quad \text { for any } m \geq 1,
$$

where $\beta_{m}=\max \left\{\left\|S_{m}\right\|,\left\|S_{m}^{-1}\right\|\right\}$ and $S_{m}$ is the regular matrix in Lemma 7 such that

$$
S_{m}^{-1} A_{m} S_{m}=\operatorname{diag}\left(\lambda_{1}^{m}, \cdots, \lambda_{n}^{m}\right) .
$$

Thus, it follows from Lemma 7 that $A_{m+1}$ has $n$ different eigenvalues $\lambda_{1}^{m+1}, \cdots, \lambda_{n}^{m+1}$.

Moreover,

$$
\begin{gathered}
\left|\lambda_{i}^{m+1}-\lambda_{j}^{m+1}\right| \geq \delta \varepsilon, \quad i \neq j, \bigotimes 1 \leq i, \otimes j \leq n, \\
\left|\lambda_{i}^{m+1}\right| \geq \delta \varepsilon, \quad i=1, \cdots, n .
\end{gathered}
$$

In fact,

$$
\begin{aligned}
\left|\lambda_{i}^{m+1}-\lambda_{j}^{m+1}\right| & \geq\left|\lambda_{i}^{1}-\lambda_{j}^{1}\right|-\sum_{l=1}^{m}\left(\left|\lambda_{i}^{l+1}-\lambda_{i}^{l}\right|+\left|\lambda_{j}^{l+1}-\lambda_{j}^{l}\right|\right) \\
& \geq\left|\lambda_{i}^{1}-\lambda_{j}^{1}\right|-2 \sum_{l=1}^{m} \| A_{l+1}-A_{l}|| \\
& \geq\left|\lambda_{i}^{1}-\lambda_{j}^{1}\right|-2 \sum_{l=1}^{m} c F_{l} \\
& \geq 2 \delta \varepsilon-2 \sum_{l=1}^{m} c F_{l} .
\end{aligned}
$$

Moreover, we have

$$
\sum_{l=1}^{m} c F_{l} \leq \sum_{l=1}^{\infty} c F_{l} \leq \sum_{m=0}^{\infty}\left(c F_{1}\right)^{2^{m}} \leq \sum_{m=1}^{\infty}\left(c F_{1}\right)^{m}=\frac{c F_{1}}{1-c F_{1}}<2 c F_{1} .
$$

Thus, if $c F_{1} \leq \min \left\{1 / 2,(1 / 4) \delta \varepsilon, \delta \varepsilon /(3 n-1) \beta_{m}^{2}\right\}$, that is, $0<\varepsilon \leq \min \left\{1, c /\|Q\|_{\rho}, c /\|Q\|_{\rho}^{2}\right\}$, then by (68), we have

$$
\left|\lambda_{i}^{m+1}-\lambda_{j}^{m+1}\right| \geq 2 \delta \varepsilon-4 \varepsilon c F_{1} \geq \delta \varepsilon, \quad i \neq j, 1 \leq n .
$$

In the same way as above, we have

$$
\left|\lambda_{i}^{m+1}\right| \geq \delta \varepsilon, \quad i=1, \cdots, n .
$$

Let $D_{*}=\cap_{m+1}^{\infty} D_{\rho_{m}}=D_{\rho / 4}$. By (69), the composition of all the changes $e^{\varepsilon^{2^{m}} P_{m}}$ converges to $\psi$ as $m \longrightarrow \infty$. Obviously,

$$
\left\|\varepsilon^{2 m} Q_{m}\right\|_{D_{*}} \leq c F_{m} \longrightarrow 0, \quad m \longrightarrow \infty .
$$

Furthermore, it follows from (71) that $A_{m}$ is convergent as $m \longrightarrow \infty$. Define $B=\lim _{m \rightarrow \infty} A_{m}$. Then, under the symplectic change of variables $x=\psi(t) y, m \longrightarrow \infty$ Hamiltonian system (1) is changed into $\dot{y}=B y$.

Now we prove that, for most sufficiently small $\varepsilon$, such symplectic transformation exists. From the above iteration, we need to prove that the nonresonant conditions

$$
\left|\langle k, \omega\rangle \sqrt{-1}-\lambda_{i}^{m+1}+\lambda_{j}^{m+1}\right| \geq \frac{\alpha_{m}}{|k|^{3 \tau}},
$$

for all $0 \neq k \in \mathbb{Z}^{r}, 1 \leq i, j \leq n, m=0,1,2, \cdots$, hold for most sufficiently small $\varepsilon$.

$$
\text { Let } \begin{aligned}
f(\varepsilon)=\langle k, \omega\rangle \sqrt{-1}-\lambda_{i}^{m+1}+\lambda_{j}^{m+1}, i \neq j, \\
O_{i j m}^{k}=\left\{\varepsilon \in\left(0, \varepsilon_{0}\right):|f(\varepsilon)|<\frac{\alpha_{m}}{|k|^{3 \tau}}\right\},
\end{aligned}
$$

where we choose

$$
\varepsilon_{0}=\min \left\{1, \frac{c}{\|Q\|_{\rho}}, \frac{c}{\|Q\|_{\rho}^{2}}\right\},
$$

such that, for $\varepsilon \in\left(0, \varepsilon_{0}\right)$, the above iteration is convergent, and

$$
\left|\frac{d f}{d \varepsilon}\right|=\left|\frac{d}{d \varepsilon}\left(\lambda_{i}^{m+1}-\lambda_{j}^{m+1}\right)\right| \geq \delta .
$$


For $\varepsilon \in\left(0, \varepsilon_{0}\right)$, by $(71)$, we have

$$
\begin{aligned}
\left\|A_{m+1}-A_{1}\right\| & \leq\left\|A_{m+1}-A_{m}\right\|+\cdots+\left\|A_{2}-A_{1}\right\| \\
& \leq c F_{m}+\cdots+c F_{2} \leq 2 c F_{1} \leq \frac{1}{2} \delta \varepsilon .
\end{aligned}
$$

Hence,

$$
\begin{aligned}
|f(\varepsilon)| \geq & \left|\langle k, \omega\rangle \sqrt{-1}-\lambda_{i}+\lambda_{j}\right|-\left|\lambda_{i}^{1}-\lambda_{i}\right| \\
& \quad-\left|\lambda_{j}^{1}-\lambda_{j}\right|-\left|\lambda_{i}^{1}-\lambda_{i}^{m+1}\right|-\left|\lambda_{j}^{1}-\lambda_{j}^{m+1}\right| \\
\geq & \frac{\alpha}{|k|^{\tau}}-2 q \varepsilon-2|| A_{m+1}-A_{1} \mid \geq \frac{\alpha}{|k|^{\tau}}-2 q \varepsilon-\delta \varepsilon \\
\geq & \frac{\alpha}{|k|^{\tau}}-3 M \varepsilon_{0},
\end{aligned}
$$

where $M=\max \{q, \delta\}$.

If $1 /|k|^{\tau}>6 M \varepsilon_{0} / \alpha$, then

$$
|f(\varepsilon)| \geq \frac{\alpha}{2|k|^{\tau}}>\frac{\alpha_{m}}{|k|^{3 \tau}}
$$

and $O_{i j m}^{k}=\varnothing$.

Suppose that $1 /|k|^{\tau}<6 M \varepsilon_{0} / \alpha$. By (83), it follows that

$$
\operatorname{meas}\left(O_{i j m}^{k}\right)<\frac{\alpha_{m}}{|k|^{3 \tau} \delta}
$$

Thus,

$$
\begin{aligned}
\operatorname{meas}\left(\bigcup_{i \neq j 0 \neq k \in \mathbb{Z}^{r}} O_{i j m}^{K}\right) & \leq \frac{n^{2} \alpha_{m}}{\delta} \sum_{|k|^{-\tau}<6 M \varepsilon_{0} / \alpha} \frac{1}{|k|^{3 \tau}} \\
& \leq \frac{n^{2} \alpha_{m}}{\delta} \cdot \frac{36 M^{2} \varepsilon_{0}^{2}}{\alpha^{2}} \sum_{k \in \mathbb{Z}^{r}} \frac{1}{|k|^{\tau}} \leq \frac{c \varepsilon_{0}^{2}}{m^{2}}
\end{aligned}
$$

Let

$E_{m}=\left\{\varepsilon \in\left(0, \varepsilon_{0}\right):\left|\langle k, \omega\rangle \sqrt{-1}-\lambda_{i}^{m+1}+\lambda_{j}^{m+1}\right|>\frac{\alpha_{m}}{|k|^{3 \tau}}, \quad 0 \neq k \in \mathbb{Z}^{r}, i \neq j\right\}$.

Then

$$
\left(0, \varepsilon_{0}\right)-E_{m}=\bigcup_{i \neq j 0 \neq k \in \mathbb{Z}^{r}} O_{i j m}^{k}
$$

Thus

$$
\operatorname{meas}\left(\left(0, \varepsilon_{0}\right)-E_{m}\right) \leq \frac{c \varepsilon_{0}^{2}}{m^{2}}
$$

Let $E_{\varepsilon_{0}}=\cap_{m=1}^{\infty} E_{m}$, then

$$
\begin{aligned}
\operatorname{meas}\left(\left(0, \varepsilon_{0}\right)-E_{\varepsilon_{0}}\right) & \leq c \varepsilon_{0}^{2}, \\
\lim _{\varepsilon_{0} \rightarrow 0} \frac{\operatorname{meas}\left(\left(0, \varepsilon_{0}\right)-E_{\varepsilon_{0}}\right)}{\varepsilon_{0}} & =0 .
\end{aligned}
$$

Therefore, $E_{\varepsilon_{0}}$ is a nonempty subset of $\left(0, \varepsilon_{0}\right)$. Thus, for $\varepsilon \in E_{\varepsilon_{0}}$, Hamiltonian system (1) is reducible, i.e., there exists a symplectic transformation $x=\psi(t) y$, which changes Hamiltonian system (1) into the Hamiltonian system $\dot{y}=B y$. Thus, Theorem 3 is proved completely.

\section{The Applications}

As an example, we apply Theorem 3 to the following Schrödinger equation

$$
\ddot{x}+\varepsilon a(t) x=0,
$$

where $a(t)$ is an analytic quasiperiodic function on $D_{\rho}$ with frequencies $\omega=\left(\omega_{1}, \cdots, \omega_{r}\right)$. Denote the average of $a(t)$ by $\bar{a}$, and suppose $\bar{a}>0$.

Let $\dot{x}=y$, then equation (93) can be rewritten in the equivalent form

$$
\dot{x}=y, \quad \dot{y}=-\varepsilon a(t) x .
$$

To apply Theorem 3, we express (94) in the form

$$
\dot{z}=(A+\varepsilon Q(t)) z
$$

where

$$
\begin{aligned}
z & =\left(\begin{array}{l}
x \\
y
\end{array}\right), \\
A & =\left(\begin{array}{ll}
0 & 1 \\
0 & 0
\end{array}\right), \\
Q & =\left(\begin{array}{cc}
0 & 0 \\
-a(t) & 0
\end{array}\right) .
\end{aligned}
$$

It is easy to see that $A$ has multiple eigenvalues $\lambda_{1}=\lambda_{2}=0$; moreover, $A+\varepsilon \bar{Q}$ has two different eigenvalues $\mu_{1}=i \sqrt{\bar{a} \varepsilon}$, $\mu_{2}=-i \sqrt{\bar{a} \varepsilon}$, where $\bar{Q}$ stands for the average of the matrix $Q(t)$ and $i=\sqrt{-1}$. It is clear that

$$
\begin{aligned}
\left|\mu_{i}\right| & =\sqrt{\bar{a}} \sqrt{\varepsilon} \geq 2 \delta \varepsilon, \quad i=1,2, \\
\left|\mu_{1}-\mu_{2}\right| & =2 \sqrt{\bar{a}} \sqrt{\varepsilon} \geq 2 \delta \varepsilon,
\end{aligned}
$$

where we choose $\delta=(1 / 2) \sqrt{\bar{a}}>0$, which is a constant independent of $\varepsilon$. Therefore, Theorem 3 can be applied. It follows from Theorem 3 that the following result holds. 
Theorem 9. Assume that $a(t)$ is an analytic quasiperiodic function on $D_{\rho}$ with frequencies $\omega=\left(\omega_{1}, \cdots, \omega_{r}\right)$, and $\bar{a}>0$. If the frequencies $\omega$ of $a(t)$ satisfy the Diophantine condition

$$
|\langle k, \omega\rangle| \geq \frac{\alpha}{|k|^{\tau}}, \quad k \in \mathbb{Z}^{r} \backslash\{0\},
$$

where $\alpha>0$ is a small constant and $\tau>r-1$.

Then, there exist some sufficiently small $\varepsilon_{0}>0$ and a nonempty Cantor subset $E_{\varepsilon_{0}} \subset\left(0, \varepsilon_{0}\right)$ with positive Lebesgue measure, such that for $\varepsilon \in E_{\varepsilon_{0}}$, system (95) is reducible. Moreover, if $\varepsilon_{0}$ is small enough, the relative measure of $E_{\varepsilon_{0}}$ in $\left(0, \varepsilon_{0}\right)$ is close to 1 .

Remark 10. From Theorem 9, it follows that equation (93) can be changed into a constant coefficient differential equation for most sufficiently small $\varepsilon>0$.

Now we want to study the Lyapunov stability of the equilibrium of equation (93), using the results obtained in Section 3. If $a(t)$ is periodic in time ( $T$ is the period), one famous stability criterion was given by Magnus and Winkler [25] for Hill's equation

$$
\ddot{x}+a(t) x=0 .
$$

That is, (100) is stable if

$$
a(t)>0, \int_{0}^{T} a(t) d t \leq \frac{4}{T},
$$

which can be shown using a Poincaré inequality. Such a stability criterion had been generalized and improved by Zhang and $\mathrm{Li}$ in [26], which now is the so-called $L^{p}$ criterion. Recently, Zhang in [27] had extended such a criterion to the linear planar Hamiltonian system

$$
\begin{aligned}
& \dot{x}=m(t) y, \\
& \dot{y}=-n(t) x,
\end{aligned}
$$

where $m(t), n(t)$ are continuous and $T$-periodic functions.

However, for quasiperiodic equation (93), the results above cannot be applied directly. Now, we obtain a result about the stability of the equilibrium of equation (93).

Theorem 11. Under the conditions of Theorem 9, the equilibrium of equation (93) is stable in the sense of Lyapunov for most sufficiently small $\varepsilon>0$.

Proof. Theorem 9 tells us that, for most sufficiently small $\varepsilon$ $\epsilon\left(0, \varepsilon_{0}\right)$, there exists an analytic quasiperiodic symplectic transformation $\mathrm{z}=\psi(t) z_{\infty}$, where $\psi(t)$ has the same frequencies as $Q(t)$, which changes (95) into the Hamiltonian system

$$
\dot{z}_{\infty}=B z_{\infty}
$$

where $B$ is a constant matrix. Moreover, from the proof of Theorem 3 in Section 3, it follows that $B$ has two different eigenvalues $\lambda_{1}^{\infty}, \lambda_{2}^{\infty}$, satisfying

$$
\begin{aligned}
&\left|\lambda_{i}^{\infty}\right| \geq \delta \varepsilon(i=1,2), \\
&\left|\lambda_{1}^{\infty}-\lambda_{2}^{\infty}\right| \geq \delta \varepsilon .
\end{aligned}
$$

Furthermore, by the proof of Theorem 3, we have

$$
\|B-(A+\varepsilon \bar{Q})\| \leq c F_{1}=O\left(\varepsilon^{2}\right) .
$$

Therefore, the two different eigenvalues of $B$ are pure imaginary and can be written in the form

$$
\lambda_{i}^{\infty}= \pm i \sqrt{b}, \quad i=1,2,
$$

where $b$ can be written in the following form

$$
b=\bar{a} \varepsilon+O\left(\varepsilon^{2}\right),
$$

which depends on $\bar{a}$ and $\varepsilon$ only.

Thus, there exists a singular symplectic matrix $S$ such that

$$
S^{-1} B S=\left(\begin{array}{cc}
i \sqrt{b} & 0 \\
0 & -i \sqrt{b}
\end{array}\right) .
$$

Let $z_{\infty}=S \tilde{z}_{\infty}$, under this symplectic transformation, system (103) is changed into

$$
\dot{\tilde{z}}_{\infty}=S^{-1} B S \tilde{z}_{\infty}=\left(\begin{array}{cc}
i \sqrt{b} & 0 \\
0 & -i \sqrt{b}
\end{array}\right) \tilde{z}_{\infty} .
$$

Hence, by an analytic quasiperiodic symplectic transformation, equation (93) is changed into

$$
\ddot{x}_{\infty}+b x_{\infty}=0 .
$$

It is easy to see that equation (110) is elliptic. Therefore, the equilibrium of equation (93) is stable in the sense of Lyapunov for most sufficiently small $\varepsilon>0$.

For the existence of quasiperiodic solution of equation (93), we have the following result.

Theorem 12. Under the conditions of Theorem 9, all solutions of equation (93) are quasiperiodic with frequencies $\Omega=$ $\left(\omega_{1}, \cdots, \omega_{r}, \sqrt{b}\right)$ for most sufficiently small $\varepsilon>0$, where $b$ is given by (107).

Proof. By Theorem 9, we know that, for most sufficiently small $\varepsilon \in\left(0, \varepsilon_{0}\right)$, there exists an analytic quasiperiodic symplectic transformation which has the same frequencies as $a(t)$; by this transformation, equation (93) is changed into (110). On the other hand, it is easy to see that all solutions of equation (110) are periodic, and the frequency of these solutions is $\sqrt{b}$. 
Thus, we only need to prove that, for most sufficiently small $\varepsilon \in\left(0, \varepsilon_{0}\right)$, the following nonresonant condition

$$
\left|k_{1} \omega_{1}+\cdots+k_{r} \omega_{r+1} \sqrt{b}\right| \geq \frac{\alpha_{0}}{|k|^{5 \tau+4}}
$$

holds for all $k=\left(k_{1}, \cdots, k_{r+1}\right) \in \mathbb{Z}^{r+1} \backslash\{0\}$, where $\alpha_{0}$ is defined in Section 3, that is, $\alpha_{0}=(1 / 2) \alpha$, and $\omega=\left(\omega_{1}, \cdots, \omega_{r}\right)$ are the frequencies of $a(t)$.

If $k_{r+1}=0$, then from the Diophantine condition (99), it follows that (111) holds.

Suppose that $k_{r+1} \neq 0$. Let $g(\varepsilon)=k_{1} \omega_{1}+\cdots+k_{r} \omega_{r}+k_{r+1}$ $\sqrt{b}$, and

$$
O_{k}=\left\{\varepsilon \in\left(0, \varepsilon_{0}\right):|g(\varepsilon)|<\frac{\alpha_{0}}{|k|^{5 \tau+4}}\right\} \text {. }
$$

It follows from the nondegeneracy condition that

$$
\left|\frac{d g}{d \varepsilon}\right|=\left|\frac{d}{d \varepsilon}\left(k_{r+1} \sqrt{b}\right)\right| \geq\left|k_{r+1}\right| \delta .
$$

By (107), we have

$$
\sqrt{b} \leq 4 \delta \sqrt{\varepsilon}
$$

From the Diophantine condition (99), it follows that

$$
\begin{aligned}
|g(\varepsilon)| & \geq \frac{\alpha}{\left(\left|k_{1}\right|+\cdots+\left|k_{r}\right|\right)}-\left|k_{r+1}\right| \sqrt{b} \geq \frac{\alpha}{\left|k_{r}\right|^{\tau}}-\left|k_{r+1}\right| \sqrt{b} \\
& \geq \frac{\alpha}{|k|^{\tau}}-\left|k_{r+1}\right| 4 \delta \sqrt{\varepsilon} \geq \frac{\alpha}{|k|^{\tau}}-4 \delta|k| \sqrt{\varepsilon_{0}} .
\end{aligned}
$$

If $1 /|k|^{\tau+1} \geq 8 \delta \sqrt{\varepsilon_{0}} / \alpha$, then

$$
|g(\varepsilon)| \geq \frac{\alpha}{2|k|^{\tau}} \geq \frac{\alpha_{0}}{|k|^{5 \tau+4}},
$$

and $O_{k}=\varnothing$. that

Suppose that $1 /|k|^{\tau+1}<8 \delta \sqrt{\varepsilon_{0}} / \alpha$, it follows from (113)

$$
\operatorname{meas}\left(O_{k}\right)<\frac{\alpha_{0}}{|k|^{5 \tau+4}\left|k_{r+1}\right| \delta} .
$$

Thus,

$$
\begin{aligned}
\operatorname{meas}\left(\bigcup_{0 \neq k \in \mathbb{Z}^{r+1}} O_{k}\right) & \leq \frac{\alpha_{0}}{\delta} \sum_{1 /|k|^{\tau+1}<8 \delta \sqrt{\varepsilon_{0}} / \alpha} \frac{1}{|k|^{5 \tau+4}\left|k_{r+1}\right|} \\
& \leq \frac{\alpha_{0}}{\delta} \frac{(8 \delta)^{4} \varepsilon_{0}^{2}}{\alpha^{4}} \sum_{k \in \mathbb{Z}^{r+1}} \frac{1}{|k|^{\tau}\left|k_{r+1}\right|} \\
& \leq c \varepsilon_{0}^{2} \sum_{0 \neq k_{r+1} \in \mathbb{Z}} \frac{1}{\left|k_{r+1}\right|^{\tau+1}} \leq c \varepsilon_{0}^{2} .
\end{aligned}
$$

Then

$$
\lim _{\varepsilon_{0} \rightarrow 0} \frac{\operatorname{meas}\left(\cup_{0 \neq k \in \mathbb{Z}^{r+1}} O_{k}\right)}{\varepsilon_{0}}=0 .
$$

Therefore, (111) holds for most sufficiently small $\varepsilon \in\left(0, \varepsilon_{0}\right)$.

Thus, all solutions of equation (93) are quasiperiodic with frequencies $\Omega=\left(\omega_{1}, \cdots, \omega_{r}, \sqrt{b}\right)$ for most sufficiently small $\varepsilon>0$.

\section{Data Availability}

There is no additional data in the manuscript, because the main result is theoretical proof.

\section{Conflicts of Interest}

The authors declare that they have no conflicts of interest.

\section{Acknowledgments}

Nina Xue was supported by the National Natural Science Foundation of China (11971059) and Doctoral Funding of Weifang University (2019BS02).

\section{References}

[1] K. J. Palmer, "On the reducibility of almost periodic systems of linear differential equations," Journal of Differential Equations, vol. 36, no. 3, pp. 374-390, 1980.

[2] R. A. Johnson and G. R. Sell, "Smoothness of spectral subbundles and reducibility of quasi-periodic linear differential systems," Journal of Differential Equations, vol. 41, no. 2, pp. 262-288, 1981.

[3] E. I. Dinaburg and Y. G. Sinai, "The one-dimensional Schrödinger equation with a quasiperiodic potential," Functional Analysis and Its Applications, vol. 9, no. 4, pp. 279-289, 1976.

[4] H. Rüssmann, "On the one-dimensional Schrödinger equation with a quasi-periodic potential," Annals of the New York Academy of Sciences, vol. 357, no. 1, pp. 90-107, 1980.

[5] L. H. Eliasson, "Floquet solutions for the 1-dimensional quasiperiodic Schrödinger equation," Communications in Mathematical Physics, vol. 146, no. 3, pp. 447-482, 1992.

[6] X. Hou and J. You, "Almost reducibility and non-perturbative reducibility of quasi-periodic linear systems," Inventiones Mathematicae, vol. 190, no. 1, pp. 209-260, 2012.

[7] A. Jorba and C. Simó, "On the reducibility of linear differential equations with quasiperiodic coefficients," Journal of Differential Equations, vol. 98, no. 1, pp. 111-124, 1992.

[8] X. Junxiang, "On the reducibility of a class of linear differential equations with quasiperiodic coefficients," Mathematika, vol. 46, no. 2, pp. 443-451, 1999.

[9] J. Li and C. Zhu, "On the reducibility of a class of finitely differentiable quasi-periodic linear systems," Journal of Mathematical Analysis and Applications, vol. 413, no. 1, pp. 69-83, 2014.

[10] A. Jorba and C. Simó, "On quasi-periodic perturbations of elliptic equilibrium points," SIAM Journal on Mathematical Analysis, vol. 27, no. 6, pp. 1704-1737, 1996. 
[11] X. Wang and J. Xu, "On the reducibility of a class of nonlinear quasi-periodic system with small perturbation parameter near zero equilibrium point," Nonlinear Analysis, vol. 69, no. 7, pp. 2318-2329, 2008.

[12] H. L. Her and J. You, "Full measure reducibility for generic one-parameter family of quasi-periodic linear systems," Journal of Dynamics and Differential Equations, vol. 20, no. 4, pp. 831-866, 2008.

[13] À. Jorba, R. Ramírez-Ros, and J. Villanueva, "Effective reducibility of quasiperiodic linear equations close to constant coefficients," SIAM Journal on Mathematical Analysis, vol. 28, no. 1, pp. 178-188, 1997.

[14] J. Li and J. Xu, "On the effective reducibility of a class of quasiperiodic Hamiltonian systems," Qualitative Theory of Dynamical Systems, vol. 14, no. 2, pp. 281-290, 2015.

[15] N. Xue and W. Zhao, "On the effective reducibility of a class of quasi-periodic linear Hamiltonian systems close to constant coefficients," Journal of Function Spaces, vol. 2018, Article ID 5189873, 7 pages, 2018.

[16] Y. N. Bibikov, "Stability of zero solutions of essentially nonlinear one degree-of-freedom Hamiltonian and reversible systems," Differential Equations, vol. 38, no. 5, pp. 609-614, 2002.

[17] B. Liu, "The stability of the equilibrium of planar Hamiltonian and reversible systems," Journal of Dynamics and Differential Equations, vol. 18, no. 4, pp. 975-990, 2006.

[18] L. Wang, B. Liu, and R. Bai, "Stability of a mixed type functional equation on multi-Banach spaces: a fixed point approach," Fixed Point Theory and Applications, vol. 2010, Article ID 283827, 9 pages, 2010.

[19] L. Wang, "Intuitionistic fuzzy stability of a quadratic functional equation," Fixed Point Theory and Applications, vol. 2010, no. 1, Article ID 107182, 7 pages, 2010.

[20] L. G. Wang and B. Liu, "The Hyers-Ulam stability of a functional equation deriving from quadratic and cubic functions in quasi- $\beta$-normed spaces," Acta Mathematica Sinica, English Series, vol. 26, no. 12, pp. 2335-2348, 2010.

[21] L. Wang and J. Li, "On the stability of a functional equation deriving from additive and quadratic functions," Advances in Difference Equations, vol. 98, 12 pages, 2012.

[22] L. G. Wang, K. P. Xu, and Q. W. Liu, "On the stability of a mixed functional equation deriving from additive, quadratic and cubic mappings," Acta Mathematica Sinica, English Series, vol. 30, no. 6, pp. 1033-1049, 2014.

[23] Y. C. Wu and Y. Q. Wang, "The stability of the elliptic equilibrium of planar quasi-periodic Hamiltonian systems," Acta Mathematica Sinica, English Series, vol. 28, no. 4, pp. 801816, 2012.

[24] C. L. Siegel and J. K. Moser, Lectures on Celestial Mechanics, Springer, 1971.

[25] W. Magnus and S. Winkler, Hill's Equation, Dover, New York, NY, USA, 1979.

[26] M. Zhang and W. Li, "A Lyapunov-type stability criterion using $L^{\alpha}$ norms," Proceedings of the American Mathematical Society, vol. 130, no. 11, pp. 3325-3334, 2002.

[27] M. Zhang, "Sobolev inequalities and ellipticity of planar linear Hamiltonian systems," Advanced Nonlinear Studies, vol. 8, no. 4, pp. 633-654, 2008. 